\title{
La ruta de Paulo Freire en Chile (1964-1969): alfabetización popular e influencias del marxismo heterodoxo*
}

Paulo Freire's route in Chile (1964-1969).

Popular Literacy and Influences of Heterodox Marxism

Percurso de Paulo Freire no Chile (1964-1969):

alfabetização popular e influências do

marxismo heterodoxo

Fabian Cabaluz ** (iD) orcid.org/0000-0003-0398-7819

Beatriz Areyuna-Ibarra*** (D) orcid.org/0000-0003-3200-6777

Para citar este artículo: Cabaluz, F. y Areyuna-Ibarra, B. (2020). La ruta de Paulo Freire en Chile (1964-1969): alfabetización popular e influencias del marxismo heterodoxo. Revista Colombiana de Educación, l(80), 291-312. https://doi.org/10.17227/rce.num80-11066

\section{(c) $(1) \Theta$}

Recibido: 27/12/2019

Evaluado: 15/04/2020 


\section{Resumen}

Este artículo de investigación pretende contribuir, desde el campo de la historia de la educación, al análisis de la experiencia del exilio vivida por el pedagogo brasilero Paulo Freire en Chile durante los años 1964 y 1969. A partir de una metodología cualitativa, de carácter hermenéutico, implementando el método de análisis de contenidos, el texto se adentra en la participación de este pedagogo en las campañas de alfabetización popular del Gobierno de Eduardo Frei Montalva, en sus influencias teóricas durante el período y los desplazamientos político-pedagógicos hacia el marxismo. En las conclusiones se sostiene que la producción pedagógica freiriana elaborada durante su exilio en Chile, muestra un importante proceso de incorporación de elementos teóricos y conceptuales provenientes del marxismo heterodoxo.

\section{Palabras clave}

exilio; reforma social; alfabetización; pedagogía crítica; marxismo

\section{Keywords}

exile, social reform, literacy, critical pedagogy, marxism

\begin{abstract}
This research article aims to contribute, from the field of the history of education, to the analysis of the exile experience lived by the Brazilian pedagogue Paulo Freire in Chile during the years 1964 and 1969. From a qualitative methodology, of a hermeneutical nature, using Content Analysis, the text delves into the participation of this pedagogue in the popular literacy campaigns of the Eduardo Frei Montalva Government, in his theoretical influences during the period and the political-pedagogical movements towards Marxism. In the conclusions, it is argued that the Freirian pedagogical production elaborated during his exile in Chile shows an important process of incorporation of theoretical and conceptua elements from heterodox Marxism.
\end{abstract}

\section{Resumo}

Este artigo de pesquisa visa contribuir, desde o campo da história da educação, para a análise da experiência de exílio vivida pelo pedagogo brasileiro Paulo Freire no Chile durante os anos de 1964 e 1969. A partir de uma metodologia qualitativa, de caráter hermenêutico e com o método de análise de conteúdo, o texto investiga a participação desse pedagogo nas campanhas de alfabetização popular do Governo de Eduardo Frei Montalva, em suas influências teóricas no período e nos deslocamentos político-pedagógicos em direção ao marxismo. Nas conclusões, argumenta-se que a produção pedagógica freiriana elaborada durante seu exílio no Chile mostra um importante processo de incorporação de elementos teóricos e conceituais do marxismo heterodoxo.

\section{Palavras-chave}

exílio, reformas sociais, alfabetização, pedagogia crítica, marxismo 
Este artículo pretende contribuir desde el campo de la historia de la educación, al análisis de la experiencia del exilio vivida por el pedagogo brasilero Paulo Freire en Chile durante los años 1964 y 1969. Para ello, el trabajo se organiza en tres apartados: en primer lugar, se detiene en comprender el contexto histórico en el que Freire llega a Chile, enfatizando en el proceso de reforma agraria y las campañas de alfabetización popular impulsadas por el Gobierno demócrata cristiano de Eduardo Frei Montalva; en segundo lugar, se analizan las influencias teóricas expresadas en un conjunto de textos redactados y publicados por el pedagogo durante su estadía en Chile, enfatizando en la incorporación de autores y textos de raigambre marxista; y finalmente, se presentan conclusiones generales que subrayan la relevancia de la experiencia del exilio de Paulo Freire en Chile para el conjunto de su obra.

Es importante agregar a modo de hipótesis que, a partir de la experiencia del exilio en Chile, la obra freiriana alimentó y se nutrió de los procesos de radicalización política vividos en el país durante la década de los sesenta, lo que en términos teóricos se expresó en la incorporación de autores y planteamientos asociados a un marxismo heterodoxo y crítico.

\section{El proceso de reforma agraria y las campañas de alfabetización popular}

Con el golpe de Estado al Gobierno de Joâo Goulart en 1964, se inició en Brasil una dictadura militar que se extendió por más de veinte años, hasta 1985. Inmediatamente perpetrado el golpe, el pedagogo brasilero Paulo Freire, entonces coordinador del Programa Nacional de Alfabetización de Brasil, fue encarcelado por un total de 75 días y luego exiliado de su país natal. Tras un brevísimo pasaje por la ciudad de La Paz, en Bolivia, Freire Ilegó a Chile en noviembre de 1964, invitado por el Gobierno demócrata cristiano de Eduardo Frei Montalva. Lo precedía la efectividad del método de alfabetización desarrollado en Brasil' y la confianza que tenían depositada en él la Iglesia católica y la Conferencia Episcopal.

1 Según Carlos Rodríguez Brandao (2009), al menos dos aspectos daban respaldo a la llegada de Paulo Freire a Chile: en primer lugar, los resultados extraordinarios de su método de alfabetización con campesinos en Brasil, el cual lograba que en tan solo 45 días aprendieran a decir y escribir palabras. Entre 1963 y 1964 se organizaron cursos de capacitación de coordinadores en casi todos los estados de Brasil. El plan para el año 1964 preveía crear 2000 círculos de cultura, para alfabetizar a cerca de 2 millones de campesinos. En este sentido, sus proyecciones eran profundamente revolucionarias; y en segundo lugar, el método de alfabetización propuesto por el pedagogo brasilero se sustentaba en una crítica radical a las concepciones ingenuas de la alfabetización. 
La invitación consistió en colaborar con la alfabetización funcional, una de las políticas impulsadas por agencias internacionales, para buscar vías de desarrollo que hicieran frente al avance del comunismo. La alfabetización funcional de adultos se consideró una política educativa economicista, pues contribuía al desarrollo del trabajo y las reformas agrarias en América Latina. Este nuevo concepto se consideró un avance respecto de modelos tradicionales de alfabetización que se venían promoviendo desde la década de los cuarenta, en los cuales el aprendizaje de los rudimentos de la lectura, la escritura y el cálculo eran suficientes para considerar a los individuos alfabetizados, aun cuando solo tuvieran una lectura balbuceante y su escritura se redujera a poder firmar su nombre. Ese modelo de alfabetización tradicional se consideró ineficaz para la participación de los sujetos en el modelo productivo.

En la Conferencia Mundial de Educación de Montreal en 1960 se entregaron orientaciones precisas acerca de la urgencia de implementar planes para eliminar el analfabetismo y avanzar hacia procesos en los que la relación entre educación y trabajo, contribuyera a aumentar el factor productivo. Se acordó que la Unesco y la onu dispusieran de medios y mecanismos para impulsar esta política entre sus países miembros. En la conferencia de Punta del Este en 1961 y la Conferencia sobre Educación y Desarrollo Económico Social en América Latina, desarrollada en Chile en 1962, entre otras, se discutió la implementación de planes de alfabetización funcional en los países del continente (Soria, 1968), entendiendo la alfabetización como un proceso integral y gradual que habilita para la vida y el trabajo:

Desde el punto de vista económico, la alfabetización funcional, tiende a dar a los adultos iletrados, los recursos personales apropiados, para trabajar, producir y consumir más y mejor, desde el punto de vista social, facilitar el paso de una cultura oral a una cultura escrita y contribuir al mejoramiento personal y del grupo y lograr un mayor grado de integración social y progreso del país. (Soria, 1968, p. 59)

La reforma agraria en conjunto con la alfabetización de adultos y la reforma a la educación formal eran de gran relevancia para el programa de Gobierno de la Revolución en Libertad y se invitó a Freire para colaborar en estos procesos. No obstante, desde su llegada, el pedagogo brasilero debió enfrentarse a las profundas contradicciones que producían tanto en la izquierda como en los cristianos comprometidos con la justicia social, las propuestas reformistas dentro del modelo capitalista (Álvarez, 2011; Donoso, 2018).

La lucha contra el capitalismo dependiente y periférico, el imperialismo estadounidense y el colonialismo estaban marcando al movimiento popular en el país, todo lo cual se veía enriquecido por las formulaciones 
de la teoría de la dependencia, la teología de la liberación, los movimientos de descolonización y la experiencia de la Revolución cubana de 1959. El ambiente político era convulsionado. Atizonada por las corrientes revolucionarias de la región, durante los años sesenta, en Chile emergió con fuerza una nueva izquierda, que se constituía en términos políticos con sectores progresistas de la Democracia Cristiana, los partidos Socialista y Comunista, el Movimiento de Acción Popular Unitaria (MAPU), la Izquierda Cristiana, y el Movimiento de Izquierda Revolucionaria (MIR) (Moulian, 2014; Valenzuela, 2014).

En el escenario nombrado, el Gobierno de Eduardo Frei Montalva impulsó y desarrolló el proceso de reforma agraria que se había iniciado en $1962,{ }^{2}$ bajo la lógica de la modernización capitalista del campo. Así, entre 1964 y 1970 se expropiaron alrededor de 1300 predios, utilizando los conductos regulares de la Ley de Reforma Agraria 15.020, y para acelerar el proceso, se creó el Instituto de Capacitación en Reforma Agraria (Icira), con la finalidad de aumentar la productividad en los asentamientos. ${ }^{3}$ El desafío de incrementar la productividad del campo requería acabar con el latifundio, mediante un proceso de redistribución de tierras; un esfuerzo estatal por incorporar maquinaria pertinente al proceso productivo (tractores, arados, etc.); una reorganización social del campesinado de acuerdo a las formas de los asentamientos y sindicatos campesinos; y una inversión en apoyo técnico, cultural y educativo a las nuevas formas productivas (Bengoa, 1987).

El proceso de reforma agraria fue paulatinamente radicalizando a la sociedad chilena. La apuesta reformista del Gobierno demócrata-cristiano fue vista como insuficiente por gran parte del campesinado, amplias movilizaciones sociales se observaron en el último periodo del mandato de Eduardo Frei:

El Gobierno de Allende se inició en noviembre de 1970, bajo una fuerte presión campesina que se había agudizado al final del gobierno de Frei, las huelgas rurales, habían pasado de 142 en 1965 a 1580 en 1970, las

2 El 15 de noviembre de 1962, durante el Gobierno de Jorge Alessandri, se promulgó la Ley de Reforma Agraria 15.020. Dicha Ley, si bien prácticamente no se aplicó lo que significó que la denominaran como "reforma de macetero"-, creó dos instituciones cruciales para el proceso posterior: el Instituto de Desarrollo Agropecuario (Indap), destinado a apoyar en términos económicos y técnicos al sector agrícola, y la Corporación de la Reforma Agraria (CORA), institución destinada a parcelar y dividir los terrenos expropiados. A pesar de sus limitaciones, esta ley marcó definitivamente la consolidación de una política reformista opuesta a los intereses de la clase terrateniente.

3 Los asentamientos, fueron considerados la organización inicial de la vida socio-económica de los predios de la reforma agraria. Desde nuestra perspectiva, resulta del todo relevante el planteamiento de José Bengoa, en el que propone comprender el asentamiento como escuela de formación campesina (Bengoa, 1987). 
tomas u ocupaciones de predios de 13 en 1965 a 456 en 1970. Dentro de éstas había una fuerte presión indígena mapuche, concentrada en las regiones de Malleco y Cautín, donde actuaba el movimiento campesino revolucionario impulsado por el MIR. (Chonchol, 2017, p. 11)

El proceso de radicalización de la sociedad chilena había comenzado varios años antes con las reformas de 1958, que establecieron la cédula única y la derogación de la ley de defensa de la democracia. Con la cédula única, los partidos de derecha dejaron de ejercer control sobre el electorado en las zonas rurales, desde entonces los partidos de izquierda y la DC, que tenían como factor programático la reforma agraria, crecieron progresivamente. Asimismo, el desarrollo y fortalecimiento de las organizaciones campesinas fueron foco de su política, fomentando federaciones y luego de la ley de sindicalización campesina, confederaciones. (Avendaño, 2017). Así entonces, la reforma agraria significó para la nueva izquierda chilena la posibilidad de romper con una larga tradición oligárquica del Estado chileno, que implicaba la derrota electoral de la derecha en las elecciones parlamentarias de 1965 y la implementación, con los mecanismos del Estado, de un proceso que permitiera terminar con el largo modelo hacendal en 1967 y a su vez la posibilidad de incorporar al campesinado a la lucha de clases del movimiento popular, todo ello agudizando los conflictos de los procesos reformistas (Bengoa, 1987).

[...] la movilización campesina empieza a mostrar objetivos que sobrepasan el proyecto burgués modernizante, y que se manifiestan siempre en conflictos locales que son únicos pero que parecen al mismo tiempo ser representativos de una progresiva maduración política del campesinado. ( Hernández, 1973, p. 50)

Las campañas de alfabetización dirigidas al campesinado y a la clase trabajadora en general, propuestas por el Gobierno chileno y la Comisión Económica para América Latina (Cepal), en el marco del proceso de reforma agraria, se Ilevaron a cabo entre 1964 y 1968. Dichas campañas ocuparon un lugar central en el programa de Eduardo Frei Montalva. Según señaló el historiador Daniel Fauré:

[...] la característica fundamental del gobierno demócrata cristiano, estaría precisamente en que pareció encontrar en el espacio educativo un elemento unificador [...]. Tarea educativa que le permitía cumplir con una parte importante de su propio proyecto ("refundando" culturalmente a la ciudadanía por medio de la aplicación de sistemas educativos extensivos y de bajo costo) e integrando, en ese proceso a los sectores más emergentes entres estos "nuevos" movimientos, a fin de generar una base social que sustentara y legitimara su mantención en el poder político. (Fauré, 2011, p. 37) 
Una de las principales diferencias que tendrían las campañas de alfabetización impulsadas durante el periodo de gobierno de Eduardo Frei con respecto a las de años anteriores consistió en que en esta ocasión las campañas fueron organizadas políticamente, diseñadas técnicamente y motorizadas por el Estado, no por instituciones privadas como en años anteriores. Es así como se impulsó la creación de la Jefatura de Planes Extraordinarios de Educación de Adultos en el Ministerio de Educación Pública y se impulsó la creación de Centros de Educación Básica y Comunitaria (Cebsco) y los Centros de Educación Media Comunitaria, (Cemco). Todo lo anterior daría cuenta de la centralidad de la alfabetización popular para el Gobierno demócrata cristiano (Fauré, 2017; Silva, 2013).

Más allá de las intenciones funcionales del sector conservador de la democracia cristiana, quienes solo concebían la alfabetización popular en lógicas técnicas y económicas, el sector progresista de dicho partido, consideraba la alfabetización como una posibilidad para incrementar los niveles de conciencia del campesinado, paso fundamental en el tránsito del latifundio al asentamiento:

[...] la juventud de la Democracia Cristiana, estaba presionando para obtener un cambio más "revolucionario", desde 1963 la JDC había sido una de las facciones más críticas del capitalismo y el individualismo. Ellos habían argumentado que un gobierno demócrata cristiano representaría a los pobres de Chile y crearía una "sociedad auténticamente humana". (Kirkendall, 2005, p. 708)

Así entonces, para las juventudes demócrata-cristianas y el ala progresista del partido, la alfabetización popular cobraba un rol relevante en el programa de la Revolución en Libertad.

Inicialmente, las campañas de alfabetización estuvieron marcadas por una impronta funcional y una lógica propia del extensionismo pedagógico; sin embargo, esta concepción duró poco, pues con la llegada de Paulo Freire las consignas originales y su sello funcionalista y extensionista fueron duramente criticadas (Freire, 2010). La idea de que la educación debía acompañar los procesos de cambio y transformación social de los sujetos chocaba con las propuestas funcionalistas, pues planteaban que los campesinos pobres, no solo debían aprender a leer y escribir para poder sufragar, administrar el asentamiento e incrementar la producción, sino que en el mismo proceso debían tomar conciencia de su propia realidad y establecer un puente entre el lenguaje y sus propias experiencias vitales (Bengoa, 1987; Fauré, 2011).

La llegada de Paulo Freire a Chile se puede explicar al menos por tres grandes aristas históricas: en primer lugar, el pedagogo brasilero sería parte de una primera oleada de exiliados brasileros que, luego del golpe de Estado de 1964, se vieron obligados a abandonar su país, y que se caracteriza por

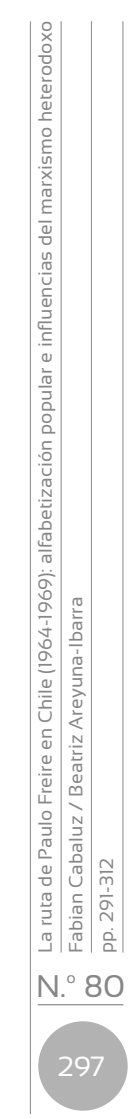


ser un grupo compuesto fundamentalmente por intelectuales y referentes políticos, entre quienes destacaron los nombres de Mario Pedrosa, Alvaro Vieira Pinto, Luiz Alberto Gomes de Souza, Paulo de Tarso, Almino Affonso, Ernani Maria Fiori, ${ }^{4}$ Plinio Arruda Sampaio y Fernando Henrique Cardoso, entre otros. ${ }^{5}$

En segundo lugar, es importante señalar que, desde su trabajo político y pedagógico en Brasil, Paulo Freire fue muy cercano al movimiento de la teología de la liberación y las comunidades eclesiales de base. Considerando lo anterior, la orden jesuita en Chile, por medio del Centro Belarmino, cumplió un papel relevante en el asilo de los brasileros en el país y particularmente en la llegada de Paulo Freire, pues tenían una gran influencia en la alta jerarquía del Partido Demócrata Cristiano y en organismos como el Instituto de Educación Rural y el Instituto Latinoamericano de Desarrollo de Estudios Sociales (Ilades). Los jesuitas eran conocedores del trabajo educativo desarrollado por el movimiento de educación de base brasilero, y por lo tanto de la propuesta de Freire.

$Y$ en tercer lugar, los personeros de gobierno vinculados particularmente al Instituto de Desarrollo Agropecuario (Indap) requerían en el marco de la reforma agraria, por un lado, avanzar en la formación de técnicos sociales, en particular jóvenes profesionales vinculados al Partido Demócrata Cristiano, que trabajaran directamente con comunidades campesinas; y por otro, capacitar a promotores campesinos, también militantes de este partido, que trabajaban directamente en la organización de asentamientos y sindicatos campesinos. Así, en noviembre de 1964, Jacques Chonchol, entonces vicepresidente de Indap, contactó a Paulo Freire, y lo vinculó como su asesor. Así se inició un riquísimo proceso educativo de la mano de la reforma agraria en el país (Silva y Andreola, 2001).

Inicialmente, desde el Indap, Paulo Freire comenzó a tensionar las lógicas de promoción popular y del extensionismo a partir de la relevancia de una educación dialógica, que promovía procesos de concientización.

4 Paulo Freire trabajó de manera muy cercana con Ernani Maria Fiori en torno a temas asociados a la cultura popular y el método de alfabetización de adultos. Compartieron su trabajo en Icira (dirigido por el brasilero Paulo de Tarso) y en numerosos espacios formativos dedicados a educadores populares y a la formación de cuadros campesinos. El trabajo mancomunado entre Freire y Fiori se tradujo en el impecable y reconocido prefacio escrito por Fiori en la Pedagogía del oprimido. Finalmente, es relevante señalar que el hijo de Ernani Maria Fiori, Ilamado José Luiz Fiori, trabajó como asistente de Paulo Freire en el Icira.

5 La llegada de los exiliados brasileros a Chile en el marco de la Revolución en Libertad fue acogida con simpatía y desconfianza por parte del Partido Demócrata Cristiano, el cual desde sus sectores más conservadores manifestó una abierta preocupación por el carácter izquierdista o revolucionario de los brasileros. Véase Silva, A. e Andreola, B. (2001), Freire e Fiori no exílio. Um projeto pedagógico-político no Chile. Editora Ritter dos Reis. 
Así, con la llegada de Freire, la alfabetización campesina y la formación de cuadros técnicos cambiaba de rumbo, girando de una concepción funcional a una popular y liberadora.

Rápidamente, a partir de sus primeros trabajos en Indap, Paulo Freire fue convocado el año 1965 como asesor y colaborador de la CORA, institución desde la cual impulsó procesos de alfabetización con campesinos en el proceso de reforma agraria. ${ }^{6}$ Ese mismo año, fue invitado por Waldemar Cortés, Jefe del Programa de Educación de Adultos del Ministerio de Educación Pública, para asesorar, capacitar y coordinar el proceso de alfabetización de adultos en sectores rurales y urbanos, a partir del método psico-social de alfabetización (Austin, 2004). Vinculado a Indap, CORA y el Programa de Educación de Adultos, Freire pudo trabajar y vincularse de manera estrecha con asentamientos, sindicatos y organizaciones campesinas, así pudo percibir en terreno el problema del analfabetismo en las zonas rurales, pero a su vez, conocer las problemáticas del analfabetismo y las altas tasas de desescolarización de la población adulta en el país.

Así entonces, desde el Indap, el CORA y el Programa de Educación de Adultos, Paulo Freire impulsó un verdadero movimiento de acción cultural, destinado a generar conciencia crítica en el campesinado que participaba del proceso de reforma agraria y en los adultos que estaban participando de programas de alfabetización, educación básica y media. Planteado en sus palabras:

Todo esto exige que la acción que se vuelca hacia el aumento de la producción en el proceso de la reforma agraria sea eminentemente cultural. Vale decir que el asentamiento, como unidad de producción, tiene que ser también una unidad cultural o de acción cultural. De acción cultural, a través de un quehacer educativo concientizador, que plantea al campesino, como problema, la manera como estuvo siendo en la estructura latifundista del silencio, y como ahora empieza a ser, en la estructura del asentamiento [...] Se hace la reforma agraria para que el hombre pueda ser más, puesto que la estructura sobre la que incide la acción transformadora no le permite sino ser menos. (Freire, 1968, p. 6)

La alfabetización popular requería un esfuerzo que sobrepasaba la capacidad del Indap, por lo que se convocó a otros actores sociales para que desempeñaran un rol de educadores en los nacientes "círculos de cultura". En esta dirección se realizaron llamados desde la Federación de Estudiantes de Chile (FECH) y desde la Central Única de Trabajadores (CUT), para lograr desarrollar desde Arica hasta Puerto Montt el proceso de alfabetización dirigido al campesinado y la clase trabajadora en su conjunto. La entusiasta

6 No deja de ser relevante mencionar que el primer manual del método psico-social de alfabetización publicado por Paulo Freire en español fue elaborado y publicado por la CORA. 
acogida de la juventud chilena a la convocatoria para impulsar la alfabetización popular se tradujo en la creación de numerosos grupos constituidos por militantes cristianos y de izquierdas, comprometidos activamente con el acompañamiento al el proceso de promoción y protagonismo popular impulsado desde las fuerzas políticas progresistas (Pinto, 2017).

La lógica de la alfabetización popular freiriana ponía en el centro del proceso al campesinado. Desde su propia experiencia de explotación, opresión y dominación debían emerger las palabras generadoras, asociadas a situaciones existenciales significativas, que organizarían el proceso de alfabetización y posalfabetización. De esta forma, se daba un giro a la alfabetización funcional y al uso de palabras-depósito que nada significaban para el/la campesino/a y su vida concreta. Planteado en palabras del pedagogo brasilero:

De esta forma, en el desarrollo de un programa de alfabetización cuyas "palabras generadoras" en lugar de ser elegidas por el educador, son, repitamos, sacadas del universo vocabular mínimo de los individuos, podemos, concomitantemente, debatir y profundizar el análisis de problemas como la reforma agraria, de la asistencia técnica, del cambio cultural, de la salud, etc. (Freire, 1968, p. 6)

Que el contenido programático del proceso de alfabetización y post-alfabetización emergiera de los propios campesinos/as, respondía también a una concepción de Paulo Freire en torno a los nacientes asentamientos campesinos, creados por la reforma agraria, al respecto Freire señaló:

El asentamiento, en la reforma agraria chilena, precisamente porque es una unidad de producción (dado que no hay producción fuera de la relación hombre-mundo) debe ser también, en su totalidad, una unidad pedagógica, en la acepción amplia del término.

Unidad pedagógica en la cual son educadores no sólo los profesores que por ventura actúen en un centro de educación básica, sino también los agrónomos, los administradores, los planificadores, los investigadores, todos los que, finalmente estén ligados al proceso. (Freire, 2010, p. 66)

También es necesario agregar que en la lógica de alfabetización freiriana resultaba fundamental modificar las anquilosadas formas de nombrar el mundo del campesinado:

Patrón; si patrón; qué puedo hacer si soy un campesino; hable, que nosotros seguimos; si el patrón lo dijo, es verdad; sabe con quién está hablando", etc., son algunas de estas palabras y expresiones incompatibles con la estructura del asentamiento, mientras esta es una estructura que se democratiza. (Freire, 1968, pp. 3-4) 
Todo lo anterior da cuenta de una comprensión humanizadora y liberadora del proceso de alfabetización.

A partir de 1967, el rumbo de la alfabetización popular y del proceso de concientización del campesinado comenzará a ser cuestionado por los sectores más conservadores del Gobierno demócrata cristiano. El énfasis del Gobierno puesto en un proceso de reforma agraria de corte productivista y económico, preocupado particularmente por el incremento de la producción agropecuaria, no coincidía con procesos sociales que apuntaban a que el asentamiento fuera un primer paso de un proceso de colectivización de la tierra y que la alfabetización fuera una herramienta cultural para la concientización campesina. Así, en la segunda mitad del mandato de Eduardo Frei Montalva se produjo un alejamiento de la política de promoción popular y un distanciamiento de los planteamientos de una educación que promoviera procesos de conciencia crítica. Los programas educativos impulsados por Indap, Cora y la Educación de Adultos del Ministerio de Educación Pública perdieron su rumbo transformador, se restringió su dinámica social, se coartaron y limitaron sus alcances y se limitó su marcha (Kirkendall, 2005).

En este escenario, el año 1968, Paulo Freire salió de Indap y de CORA, decepcionado por el giro político de los organismos, y se vinculó al Instituto de Capacitación e Investigación, Icira, un brazo de la Organización para la Agricultura y la Alimentación de las Naciones Unidas, FAO. Aunque Icira fue un organismo que operaba en conjunto con el Gobierno chileno, Freire pudo trabajar con mayor grado de independencia y dispuso de más tiempo para reflexionar y escribir sobre lo que él y sus compañeros estaban haciendo. Así entonces, desde Icira, institución en la que trabajó entre enero de 1968 y abril de 1969, Paulo Freire logró, junto a un relevante equipo de profesionales, ${ }^{7}$ sistematizar la experiencia político-pedagógica desarrollada en el marco de su exilio en Chile.

Desde Icira, Paulo Freire hizo algunas críticas a las nuevas orientaciones dadas al proceso de reforma agraria y a la alfabetización popular. Advirtió particularmente acerca del avance de los aspectos técnicos sobre lo cultural, que dejaban a la acción y la experiencia campesina a merced de una mirada mecanicista. Planteado en sus propias palabras:

Se olvida, de esta forma, que las técnicas como el saber científico, así como el procedimiento empírico de los campesinos, se encuentran condicionados histórico-culturalmente. En ese sentido son manifestaciones culturales ante las técnicas de los especialistas, como el procedimiento

7 El equipo de profesionales que trabajó con Paulo Freire en Icira estuvo conformado por Marcela Gajardo, José Luiz Fiori (hijo de Ernani Maria Fiori), María Edy Ferrera, Sergio Villegas, Odilio Friedrich, Margarita Depetris, Maria Helena de Jordán y Martina Huges. 
empírico de los campesinos. Este procedimiento se halla en un marco cultural, que no debe sencillamente ser "invadido" por manifestaciones de otro marco, en este caso, las técnicas de los especialistas. Es un error científico y una falta de humildad subestimar la capacidad creadora y recreadora de los campesinos, despreciar sus conocimientos, aun cuando a nivel mágico o de la pura "doxa", o intentar llenarlos de lo que nos parece lo cierto. (Freire, 1968, pp. 2-3)

Desde finales de 1968, el carácter conservador y proestadounidense de la juventud demócrata cristiana que vivía el proceso de la Revolución en Libertad, desató profundos debates y cuestionamientos en la juventud chilena radicalizada, que tuvieron su corolario en marzo de 1969, cuando el ministro del Interior, Edmundo Pérez Zujovic mandó a reprimir a pobladores que ocuparon un terreno en un sector de Puerto Montt Ilamado Pampa Irigoin con el objeto de lograr su expropiación y construir allí sus futuros hogares. El hecho desencadenó el asesinato de diez pobladores y dejó setenta heridos, lo que produjo tensiones irreconciliables en el seno de la democracia cristiana. Dos meses después de la matanza de Pampa Irigoin, en mayo de 1969, se produjo la salida de sectores de izquierda del Partido Demócrata Cristiano y la fundación del Movimiento de Acción Popular Unitario, MAPU. En este escenario, los sectores conservadores presionaron para sacar a los izquierdistas del partido, y el pedagogo brasilero era considerado uno de ellos.

Los sectores conservadores del Partido Demócrata Cristiano acusaron a Paulo Freire de marxista y de promover con sus ideas pedagógicas la lucha de clases en el campesinado y los sectores populares. Tales acusaciones condujeron a que no se le renovara su contrato de trabajo, y a su consiguiente decisión de salir del país, aceptando una invitación cursada por la Universidad de Harvard. Así, en abril de 1969, Paulo Freire salió de Chile, dejando un tremendo legado para el campo educativo y pedagógico nacional (Silva y Andreola, 2001).

A continuación, nos detendremos en las influencias teóricas y en los desplazamientos político-pedagógicos del pedagogo brasilero durante su estadía en Chile.

\section{Influencias teóricas y desplazamientos político-pedagógicos}

Durante los cinco años que Paulo Freire vivió en Chile, entre noviembre de 1964 y abril de 1969, produjo y publicó libros, artículos, informes y textos de gran relevancia para el campo pedagógico latinoamericano y mundial. Dicho corpus bibliográfico se encuentra conformado por los siguientes textos: 
» La primera publicación del libro La educación como práctica de la libertad, en la primavera de $1965 .^{8}$

» Los ocho textos producidos por Freire en Icira entre 1968 y 1969 , y publicados en 1971 en el libro titulado Sobre la acción cultural. ${ }^{9}$ Los textos fueron: "La alfabetización de adultos", "El rol del trabajador social en el proceso de cambio", "Investigación de la temática generadora", "A propósito del tema generador y del universo temático", "La concepción bancaria de la educación y la deshumanización", "La concepción problematizadora y la humanización", "El campesino también puede ser autor de sus propios textos de lectura" y "La práctica del método psicosocial".

» El libro titulado ¿Extensión o comunicación? La concientización en el medio rural, ${ }^{10}$ elaborado a comienzos del año 1968, inscrito en 1969 y publicado por primera vez en noviembre de 1971.

» Los dos informes presentados como asesor de la Unesco en 1968, bajo los títulos de La alfabetización funcional en Chile y Acción cultural y concientización.

» Los cuatro textos publicados por Icira, disponibles en el archivo José María Arguedas de la Biblioteca Nacional de Chile: El compromiso del profesional con la sociedad (1968), El asentamiento como una totalidad (1968), Acción cultural y reforma agraria (1968) y Desarrollo de la comunidad.

» Los artículos "Dialogicidad de la educación" y "Consideraciones críticas en torno al acto de estudiar" publicados en la revista Pastoral Popular (1968/1969, n.os 107 y 110, respectivamente) del Centro Ecuménico Diego de Medellín.

» El texto Papel da educação na humanização, publicado en octubre de 1969, que contiene una síntesis de charlas presentadas por Paulo Freire en mayo de 1967 en la ciudad de Santiago de Chile.

» El primer manuscrito del libro Pedagogía del oprimido, entregado como obsequio a Jacques Chonchol y su esposa María Edy, antes de partir de Chile. Dicho texto fue donado por Jacques Chonchol a la Biblioteca Nacional de Brasil en el 2014. Una edición facsímil del manuscrito fue publicada por el Instituto Paulo Freire y la

8 La primera edición en portugués de dicho texto del año 1967 fue introducida por Francisco Weffort; la primera edición uruguaya de 1969 fue presentada por Julio Barreiro, nombre de pila empleado en el marco de la dictadura militar brasilera por su compañero y amigo, el educador popular Carlos Rodríguez Brandao.

9 El trabajo de ordenamiento y edición de los textos fue responsabilidad de la pedagoga chilena Marcela Gajardo, quien trabajó en Icira junto a Paulo Freire.

10 Libro editado por Marcela Gajardo y Jorge Mellado, ambos integrantes de Icira; y presentado por Antonio Corvalán, Director Nacional de Icira, Solón Barraclough, director internacional de Icira y Jacques Chonchol. 
Universidad Nueve de Julio de San Pablo. En Chile, una coedición del Programa Interdisciplinario de Investigaciones en Educación y la Universidad Tecnológica Metropolitana hizo posible la publicación en 2018 del manuscrito original.

A partir de la reconstrucción del corpus bibliográfico producido y publicado por Paulo Freire durante su estadía en Chile, nos interesa profundizar en las diferentes influencias teóricas que van emergiendo en sus textos, las cuales van avanzando con el pasar de los años desde la fenomenología y el existencialismo hacia una creciente incorporación de referencias marxistas. De hecho, a modo de hipótesis, se ha sostenido que el pensamiento político-pedagógico freiriano, a partir de la experiencia del exilio en Chile, se nutre de la radicalización política vivida en el país, lo que en términos teóricos se traduce en una creciente incorporación de autores y planteamientos de raigambre marxista. Pasemos entonces a analizar las diferentes influencias del pensamiento freiriano durante 1964 y 1969.

Con respecto a las influencias teóricas, es importante enfatizar en que los textos producidos en Chile poseen una importante referencia a autores asociados al existencialismo y la fenomenología, entre los que se destacan particularmente Karl Jasper, Edmund Husserl, Emmanuel Mounier, Jean Paul Sartre, ${ }^{11}$ Merleau Ponty ${ }^{12}$ y Eduardo Nicol. ${ }^{13}$ Uno de los principales argumentos desarrollados a partir de esta influencia es aquel que enfatiza en el carácter inacabado e inconcluso del ser humano, en su historicidad, todo lo cual permite enfatizar en la relevancia de la transformación del mundo y de las relaciones sociales, que se revelan como posibilidades para su humanización. A su vez, todo el abordaje que realizó el pedagogo brasilero en torno al concepto de cultura alude permanentemente a las nociones de situaciones y experiencias existenciales, todo lo cual contiene una profunda carga de estas corrientes filosóficas. A lo anterior, habría que agregar que las reflexiones sobre la relación entre subjetividad y objetividad, entre conciencia y mundo, entre lo pensado y lo vivido, también se inspiran en el existencialismo y la fenomenología (Andreola, 2015).

Otra influencia que comienza a emerger en la obra freiriana durante su exilio en Chile es la de dos referentes del pensamiento anticolonial: Franz Fanon y su libro Los condenados de la tierra y Albert Memmi con El colonizador y el colonizado. Ambos autores son retomados para tratar el problema

11 En la Pedagogía del oprimido, Freire se refirió a Jean Paul Sartre y su texto El hombre y las cosas, abordando la idea del saber como concepción digestiva o alimenticia, lo que fue del todo influyente en su conceptualización sobre la educación bancaria.

12 Para reflexionar en torno a la categoría de dialogicidad, Paulo Freire referenciará un texto de Merleau Ponty titulado Fenomenología de la percepción. Véase Freire (1971b).

13 Filósofo catalán exiliado en México luego de la guerra civil española, fundador del Instituto de Investigaciones Filosóficas de la Universidad Nacional Autónoma de México. Freire retomó planteamientos de Nicol centrados en el conocimiento, la relación comunicativa y el acto dialógico. Véase Freire (2010). 
de la colonización, la relación entre colonizador y colonizado, la violencia ejercida por parte del opresor, la conciencia colonizada y la introyección del opresor en la subjetividad del oprimido (Freire, 2009 y 2012).

Un comentario requiere detenerse en los educadores y filósofos brasileros referenciados y citados por Paulo Freire, pues son nombres que emergen de manera recurrente en sus textos. Entre dichos autores es importante mencionar a su maestro Álvaro Viera Pinto y su libro Conciencia y realidad nacional, el cual inspira sus reflexiones sobre el analfabetismo y la noción de "conciencia intransitiva" que venía siendo trabajado por Freire desde su tesis doctoral publicada bajo el título Educación y actualidad brasilera (Freire, 1971d, 1971e, 2009 y 2012); a su colega y amigo Ernani María Fiori, cuyo texto Concientización y educación retomó, pues planteó precisiones al método de alfabetización freiriano, específicamente al momento introductorio en que se reflexiona en torno al concepto de cultura (Beca et al., 2013; Freire, 1971f); y a Anísio Teixeira, referente del movimiento de pioneros de la escuela nueva en Brasil, y su texto Revolución y educación, desde donde sustenta el desprecio de las élites latinoamericanas por la educación de las clases dominadas (Freire, 2009).

A partir de la hipótesis sostenida en este trabajo, y de acuerdo con los textos producidos por Paulo Freire en Chile, hemos podido constatar que existen al menos cinco afluentes teóricos de raigambre marxista que son incorporados en la obra del pedagogo brasilero.

Como primer afluente teórico se debe señalar que, paulatinamente, van calando en las reflexiones freirianas algunos planteamientos de la Escuela de Frankfurt, en particular las contribuciones de Erich Fromm y Herbert Marcuse. La influencia del primero de ellos, psicoanalista freudiano y marxista, se dio a partir de los textos Miedo a la libertad, El corazón del hombre y Marx y su concepto de hombre, que recuperó principalmente para profundizar en el concepto de cosificación del ser humano y en el problema de la alienación del lenguaje (Freire, 2009). Por su parte, la influencia de Herbert Marcuse se expresa en las referencias a los textos $E I$ hombre unidimensional, Eros y civilización, los cuales utilizó para referirse a las formas de control social desplegados por los opresores. Finalmente, es importante resaltar que los textos de Fromm y Marcuse eran estudiados por los integrantes del Icira, e influenciaron las categorías de Invasión cultural y Síntesis cultural elaboradas en el marco del proceso de alfabetización de campesinos (Freire, 1971a y 2012).

Como segundo afluente, podemos constatar que fueron incorporándose referencias y citas de marxistas críticos o heterodoxos, es decir que polemizaban con la perspectiva ortodoxa promovida desde la URSS, el marxismo-leninismo y el materialismo dialéctico. Entre ellos, Paulo Freire retomó la obra canónica de Gyorgy Lukacs titulada Historia y conciencia de clases. Dicho texto fue referenciado por primera vez en la Pedagogía

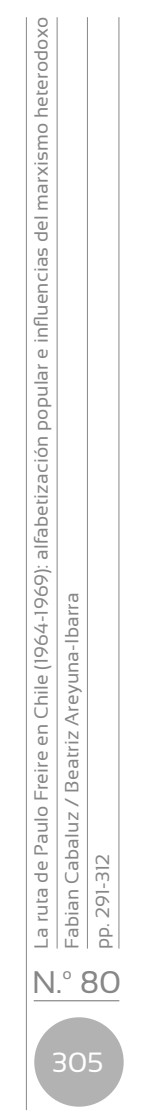


del oprimido, para referirse al problema de la conciencia y al proceso de concientización, sin embargo en un texto anterior, del año 1968, titulado La concepción bancaria y la concepción problematizadora de la educación, al hablar sobre la concientización en su método de alfabetización, había referido a las nociones de "conciencia de sí" y "conciencia para sí" y a la relación dialéctica entre humanización y deshumanización, pero sin hacer referencia al filósofo húngaro (Freire, 2012).

En sintonía con lo anterior, Paulo Freire fue incorporando referencias y citas de otros cuatro marxistas que se sitúan en la vereda de la heterodoxia: al filósofo checo Karel Kosik y su libro La dialéctica de lo concreto; a los filósofos franceses Lucien Goldman con su libro Las ciencias humanas y la filosofía y Louis Althusser con su libro La revolución teórica de Marx; y al filósofo yugoeslavo Gajo Petrovic y su texto titulado Hombre y libertad. La referencia a Karel Kosik se dio en función de profundizar en la categoría de praxis, entendiéndola como actividad humana que permitía la transformación y creación del mundo, la producción de objetos, cosas sensibles, instituciones sociales, ideas, etc. (Freire, 1971d y 2012). Con respecto a la referencia a la obra de Lucien Goldmann, Paulo Freire retomó las nociones de "conciencia real" o conciencia efectiva que se encuentra limitada por los obstáculos de la realidad concreta, y la "conciencia máxima posible", lo que se asemeja a un horizonte de posibilidad que se va descubriendo y concretando en la acción humana transformadora, noción de la que surge la categoría freiriana de "inédito viable"; y en lo relativo a la obra de Louis Althusser, los textos producidos por Freire, relevaron la noción de "sobredeterminación", para profundizar en la relación entre la infraestructura y la superestructura (Freire, 1971c y 2012). Finalmente, con relación a la referencia a Gajo Petrovic, referente intelectual y político del Grupo Praxis de Yugoslavia, ícono del marxismo herético y la filosofía de la praxis de la segunda mitad del siglo xx, en la Pedagogía del oprimido, Freire lo referencia y cita para profundizar en torno a la praxis dialógica y en la relevancia de la dialogicidad para un proyecto histórico revolucionario (Freire, 1971c y 2012).

Como tercer afluente, y ya entrando a exclusivamente a las referencias señaladas en la Pedagogía del oprimido, se puede reconocer una lectura de planteamientos formulados por referentes políticos del marxismo latinoamericano, tales como a Ernesto "Che" Guevara, y sus textos El diario del Che en Bolivia y Relatos de la guerra revolucionaria, así como de algunos discursos de Fidel Castro. Ambos referentes fueron citados por el pedagogo brasilero para referirse a la centralidad del amor en la praxis revolucionaria; a la introyección del opresor en las clases oprimidas (campesinos y obreros); y a la relevancia de la dialogicidad entre el liderazgo revolucionario y el pueblo oprimido (Freire, 2012). 
Un cuarto afluente nos remite a las referencias realizadas por Paulo Freire con respecto a Lenin y Mao Zedong. Del líder revolucionario bolchevique, Freire recogió planteamientos del libro ¿Qué hacer?, para reafirmar la noción de praxis y problematizar las posiciones que consagraban distanciamientos entre teoría y práctica en los procesos revolucionarios. Es decir, en la crítica freiriana al verbalismo, que denostaba la acción transformadora y al activismo que restaba relevancia a la teoría revolucionaria, se encuentran los planteamientos de Lenin. Y con respecto a la obra de Mao Zedong, Freire referencia El frente unido en el trabajo cultural para oponerse al vanguardismo y el verticalismo de cierta izquierda revolucionaria que actúa intentando hegemonizar y direccionar al pueblo, más que comprometerse en un proceso de lucha de liberación junto al pueblo. Así, afirmándose en el líder revolucionario chino, reivindicó proyectos revolucionarios que emergían de las necesidades reales de las masas y de sus deseos y anhelos libremente expresados. Además, retomó un fragmento donde Mao se refiere a la importancia de la dialogicidad en la constitución del contenido programático de la educación. "Sabe qué es lo que mantengo desde hace algún tiempo, es necesario enseñar a las masas con precisión lo que hemos recibido de ellas con confusión" (Freire, 2012, p. 89).

Y el quinto afluente se asocia directamente a las referencias que realizará el pedagogo brasilero con respecto a la obra de Karl Marx. La primera referencia que encontramos en los textos producidos en Chile nos remite a ¿Extensión o comunicación? La concientización del medio rural, pues allí retomó la tercera de las Tesis sobre Feuerbach, para polemizar con el "objetivismo acrítico y mecanicista", que sin decirlo explícitamente remitía a las concepciones dogmáticas y economicistas del marxismo. A partir de la tercera tesis, Freire reflexiona sobre la complejidad de la relación entre sujeto y estructura, o si se prefiere, a la relación entre subjetividad y objetividad (Freire, 2010). En un segundo momento, ya en la Pedagogía del oprimido vuelven a aparecer referencias y citas en torno a la obra del filósofo de Tréveris, en particular a La sagrada familia y otros escritos, para aludir a la concientización; las Tesis sobre Feuerbach para reflexionar en torno a la relación entre las circunstancias y los sujetos; y los Manuscritos de 1884. Economía, política y filosofía para enfatizar en el carácter histórico y transformador del ser humano, como ser de la praxis (Freire, 2012).

\section{Conclusiones}

En consideración de la participación del pedagogo brasilero en la orientación político-pedagógica de las campañas de alfabetización y en diversas instancias educativas asociadas a la reforma agraria, parece del todo pertinente sostener que la ruta de Paulo Freire en Chile marcó un importante giro en la 
concepción de la alfabetización funcional sostenida por el Gobierno demócrata cristiano. El proceso de radicalización política que vivía el país era tierra fértil para una concepción educativa centrada en la concientización, la dialogicidad y la liberación. La concepción político-pedagógica freiriana fue adoptada por convicción por un grupo relevante de educadores del país, quienes siguieron por un largo periodo, por lo menos hasta la década de los noventa, alimentando las luchas por democratizar y transformar la educación y las prácticas educativas desde las comunidades y los territorios.

A partir de la información levantada en torno a las influencias teóricas de Paulo Freire durante los cinco años que estuvo en Chile, no resulta aventurado sostener que, al calor de la experiencia social compartida en este país, el pedagogo brasilero radicalizó numerosos planteamientos de sus argumentos e incorporó un número creciente de referentes marxistas a sus construcciones conceptuales. Evidentemente, el marxismo del que se alimentó Paulo Freire fue aquel que provenía de una vertiente lejana a la ortodoxia, el positivismo, economicismo y mecanicismo que circulaba por numerosos manuales elaborados o autorizados por la unión soviética. En este sentido, el pedagogo brasilero alimentó su obra de la corriente cálida del marxismo, de versiones heterodoxas y heréticas, las cuales traducidas a las problemáticas reales y concretas que vivían las clases oprimidas del campo y la ciudad en Brasil, Chile y América Latina, servían para nutrir y robustecer sus análisis y propuestas político-pedagógicas. ${ }^{14}$

El proceso de politización que vivió la sociedad chilena durante la década de los sesenta — signado por los debates en torno a la reforma agraria, la nacionalización de los recursos naturales estratégicos, la preocupación por fortalecer la soberanía nacional restando poder a las fuerzas del imperialismo estadounidense, las propuestas discursivas y prácticas que abogaban por la construcción de poder popular, la relevancia de impulsar procesos de alfabetización popular que mejoraran las condiciones objetivas de los sectores sociales marginados y favorecieran las condiciones subjetivas para impulsar procesos revolucionarios-indiscutiblemente influyeron en la obra de Paulo Freire. De hecho, en numerosas ocasiones, el pedagogo brasilero se refirió a la relevancia del trabajo desarrollado en Chile para la maduración de la pedagogía de la liberación. ${ }^{15}$ En este

14 Una pregunta que queda abierta refiere a la relación entre Paulo Freire y la obra del marxista italiano Antonio Gramsci, pues de acuerdo a la revisión del corpus bibliográfico producido en Chile no existe ninguna referencia explícita a uno de los mayores exponentes de la filosofía de la praxis. Tal vez se pueda aventurar como hipótesis que durante la década de los sesenta, Paulo Freire no logró acceder a traducciones al portugués o el castellano de las obras gramscianas, pero sin duda, esto daría lugar a otro trabajo.

15 El educador chileno, Guillermo Williamson sostuvo que Paulo Freire reconoció en numerosos pasajes de su obra que su paso por Chile contribuyó en la radicalización de su pensamiento, puesto que la sociedad chilena de los sesenta se encontraba altamente politizada. Véase Williamson (2001). 
sentido, creemos, que el ejercicio de análisis de las influencias teóricas de Paulo Freire durante el periodo de 1964-1969, da cuenta de un desplazamiento de su pensamiento político-pedagógico hacia la izquierda, incorporando cada vez más a referentes del marxismo heterodoxo.

Lo anterior no implica desconocer los planteamientos del propio Paulo Freire, quien matizó reiteradas veces su relación con Marx y el marxismo. De hecho, en una entrevista realizada por Ligia Chiappini en 1979, ante la pregunta si se reconocía como marxista, Freire respondió:

Indiscutiblemente, fui en mi juventud hacia el campesino y al obrero de mi ciudad, movido por mi opción cristiana. De la cual no reniego. Viendo eso, el dramatismo existencial de los hombres y de las mujeres con quienes comencé a dialogar me remitió a Marx. Es como si los campesinos y los obreros me hubieran dicho "Mira Paulo, ven acá ¿Conoces a Marx?" [...]. No quiero decir que hoy soy un "experto" en Marx, o que soy un marxista, Hasta por un problema de humildad. Yo creo que es muy serio decir de alguien que es marxista [...]. No fui a las clases oprimidas por causa de Marx. Fui a Marx por causa de ellas. (Gadotti, 2001, p. 613)

Lo anterior lo reafirmó en una conversación desarrollada con Adriano Nogueira y Dermeval Saviani meses antes de su muerte, en la cual sostuvo:

Mira qué interesante. Me preguntaron, recientemente en un debate: "Paulo, ¿̇ú te definirías como marxista?". Y yo comentaba: "Yo les digo que, por respeto a Marx, no me defino marxista". Un teórico que acepte algún a priori de la Historia o en la Historia no es marxista [...]. Igualmente, si yo acepto a Dios como un a priori y no admito escuchar preguntas sobre ¿Cómo es éste Dios? ¿Cómo obra? ¿Él es hombre, es mujer o es un fluido? ¿Él vive aquí o allá?... Si no supiese explicar esto históricamente no estaría siendo marxista". (Saviani, 2010, pp. 9-10)

Como se puede observar en las dos citas, Paulo Freire, no se definió como marxista, sin embargo, jamás negó su vinculación con la obra de Marx y con el marxismo. Como hemos podido constatar en nuestra investigación, la incorporación sistemática de referentes teóricos e intelectuales marxistas se desarrolló desde el exilio en Chile, momento a partir del cual resulta innegable la existencia de una veta marxista en su obra.

\section{Referencias}

Álvarez, A. (2011). Influencia del pensamiento de Paulo Freire en Chile: ideas para un debate. Perspectivas, 22, 215-237.

Andreola, B. (2015). Existencia. En D. Streck, E. Redín, J. Zitkoski (orgs.). Diccionario Paulo Freire. Ceaal. 
Austin, R. (ed.). (2004). Diálogos sobre Estado y educación popular en Chile: de Frei a Frei (1964-1993). Ediciones Cecatp.

Avendaño, O. (2017). La gradualidad de la reforma agraria chilena, el papel que jugaron los partidos políticos. En Reforma Agraria, Dossier Le monde Diplomatique (pp. 23-28). Ediciones Aún Creemos en los Sueños.

Beca, C., Richards, C. y Bianchetti, L. (2013). Ernani Maria Fiori: un profesor brasilero en tierras chilenas testimonios. Educação \& Realidade, 38(3), 1021-1034.

Bengoa, J. (1987). Educación campesina y reforma agraria en Chile. Proposiciones, 15, 162-173.

Donoso, A. (2018). La educación en las luchas revolucionarias. Iván Illich, Paulo Freire, Ernesto Guevara y el pensamiento latinoamericano. Editorial Quimantú.

Chonchol, J. (2017). Reforma Agraria, la revolución chilena en el campo. Reforma Agraria, Dossier Le Monde Diplomatique (pp. 5-16). Ediciones, Aún Creemos en los Sueños.

Fauré, D. (2011). Auge y caída de la educación popular chilena (1964-1994) (tesis de magíster), Universidad de Santiago de Chile.

Fauré, D. (2017). Entre Roger Vekemans y Paulo Freire: las campañas de alfabetización de adultos en el gobierno de Eduardo Frei (1964-1970). Kavilando, 9(1), 51-72.

Freire, P. (1968). La alfabetización funcional en Chile. Instituto de Capacitación e Investigación en Reforma Agraria.

Freire, P. (1971a). Sobre la acción cultural. Icira.

Freire, P. (1971b). Investigación de la temática generadora. Sobre la acción cultural. Icira.

Freire, P. (1971c). Acción cultural y reforma agraria. Sobre la acción cultural. Chile, Icira.

Freire, P (1971d). A propósito del tema generador y del universo temático. Sobre la acción cultural. Icira.

Freire, P (1971e). La alfabetización de adultos. Sobre la acción cultural. Icira.

Freire, P. (1971f). La práctica del método psico-social. Sobre la acción cultural. Chile, Icira.

Freire, P. (2009). La educación como práctica de la libertad. Siglo xxı.

Freire, P. (2010). ¿Extensión o comunicación? La concientización del medio rural. Siglo xxı Editores.

Freire, P. (2012). Pedagogía del oprimido. Siglo xxı.

Gadotti, M. y Torres, C. (comp.) (2001). Paulo Freire: una biobibliografía. Siglo XXI. 
Hernández, S. (1973). El desarrollo capitalista del campo chileno. Sociedad y Desarrollo 30.

Kirkendall, A. (2005). Paulo Freire, Eduardo Frei, Literacy Training and the Politics of Consciousness Raising in Chile, 1964 to 1970. Journal of Latin American Studies, 36(4). http://www.jstor.org/stable/3875536?se$\mathrm{q}=1$ \#page_scan_tab_contents

Pinto, R. (2004). Paulo Freire: un educador humanista cristiano en Chile. Pensamiento Educativo. Revista de Investigación Educacional Latinoamericana, 34, 234-258.

Pinto, R. (2017). Más allá de medio siglo de conversaciones con Paulo Freire. Paulo Freire. Revista de Pedagogía Crítica, 15(17), 69-98.

Rodríguez Brandao, C. (2009). Educación y concienciación. En P. Freire, La educación como práctica de la libertad. Siglo xxı.

Saviani, D. (2010). Educación. Preparación para el siglo xxı (Diálogo con Paulo Freire y Adriano Nogueira). Interlocuciones pedagógicas. Autores Associados.

Silva, A. y Andreola, B. (2001). Freire e Fiori no exílio. Um projeto pedagógico-político no Chile. Editora Ritter dos Reis.

Silva, C. y Pérez, C. (2013). Educación y proyectos desarrollistas: discursos y prácticas de alfabetización popular en Chile, 1960-1970. Cuadernos Chilenos de Historia de la Educación, 1, 87-111.

Soria, L. (1968). Alfabetización funcional de adultos. Centro de Desarrollo para la Educación de la Comunidad en América Latina.

Valenzuela, E (2014). Dios, Marx... y el MAPU. LOM Ediciones.

Williamson, G. (2001). Paulo Freire: 1964-1969: su paso por Chile y el Chile por el cual pasó. En M. Gadotti y C. Torres (comps.), Paulo Freire: una biobibliografía (pp. 163-165). Siglo xxı. 